\title{
Effects of Atrial Fibrillation on the Outcome of the Rehabilitation in Patients With Cerebral Infarction
}

\author{
Ja-Young Kim, MD, Su-Jin Lee, MD, Jin-Hong Kim, MD, Cheol-Min Choi, MD, \\ Seo-Ra Yoon, MD, Kwang-Ik Jung, MD
}

Department of Rehabilitation Medicine, Gwangju Veterans Hospital, Gwangju, Korea

\begin{abstract}
Objective To evaluate the influence of atrial fibrillation (Af) on the clinical characteristics and rehabilitation outcomes of patients with cerebral infarction.

Methods We evaluated 87 of 101 consecutive patients with cerebral infarction admitted to the department of physical medicine and rehabilitation during their rehabilitation period. The patients were divided into two groups, Af and non-Af groups. We estimated characteristics of patient demographic features, disease duration, length of hospital stay, other comorbidities and risk factors for stroke, and functional status at admission and at discharge and compared those in patients with and without Af. Functional Independence Measure (FIM), the Modified Barthel Index (MBI), and the PULSES profile (PULSES) were used to evaluate functional status.

Results The number in the Af group was 20 (22.9\%) and that of the non-Af group was 67 (77.1\%). Demographic features, other comorbidities, motor function, cognitive function, neurological scales, and brain lesions did not differ significantly between the groups. The incidence of coronary artery disease and valvular heart disease were significantly correlated with the incidence of Af in multivariate analysis. Based on FIM, MBI, and PULSES scores, functional improvement in the Af group after rehabilitation was significantly less than that of the non-Af group.

Conclusion Af was shown to be associated with a markedly negative result in rehabilitation in patients with cerebral infarction. Thus, early recognition and proper treatment of Af may help patients achieve more effective rehabilitation.
\end{abstract}

Keywords Atrial fibrillation, Cerebral infarction, Rehabilitation outcomes

Received December 2, 2013; Accepted July 25, 2014

Corresponding author: Seo-Ra Yoon

Department of Rehabilitation Medicine, Gwangju Veterans Hospital, 99 Cheomdanwolbong-ro, Gwangsan-gu, Gwangju 506-705, Korea

Tel: +82-62-602-6295, Fax: +82-62-602-6990,E-mail: standupmd@ hanmail.net

(c) This is an open-access article distributed under the terms of the Creative Commons Attribution Non-Commercial License (http://creativecommons. org/licenses/by-nc/3.0) which permits unrestricted noncommercial use, distribution, and reproduction in any medium, provided the original work is properly cited.

Copyright ( 2014 by Korean Academy of Rehabilitation Medicine

\section{INTRODUCTION}

Atrial fibrillation is the most common arrhythmia in the elderly. In particular, atrial fibrillation has considerable morbidity and mortality rates in elderly patients and is considered a major risk factor for stroke [1]. The prevalence of atrial fibrillation is about $1 \%$ of the general population and doubles every 10 years, for example, increasing from $0.5 \%$ in people in 50 s to almost $9 \%$ in 80 s $[2,3]$.

Patients with atrial fibrillation are more at risk for stroke than patients with sinus rhythm $[4,5]$. Go [6] reported 
that atrial fibrillation accounted for $20 \%$ of all occurrences of cerebral infarction. Wolf et al. [7] also revealed that patients with chronic atrial fibrillation without valvular heart disease or structural heart disease had a 5 times higher prevalence of stroke while patients with valvular heart disease or structural heart disease along with chronic atrial fibrillation had a 17 times higher prevalence of stroke. It has been reported that from 9.3\% to $23 \%$ of patients with stroke have atrial fibrillation [8-10].

The role of atrial fibrillation as a prognostic factor in stroke has not been studied thoroughly. However, most previous studies reported that atrial fibrillation was related to more frequent stroke recurrence and a higher fatality rate $[8,11,12]$. Fisher [13] reported that patients with stroke and atrial fibrillation had more severe and fatal stroke symptoms more commonly than patients with stroke without atrial fibrillation, although recent study results were not consistent with this $[11,14]$.

It is also not yet clear how atrial fibrillation affects the process of recovery from stroke. The most serious concern of rehabilitation professionals is the effect of atrial fibrillation on functional and clinical outcomes and longterm follow-up of patients with stroke. Until recently, few studies had examined rehabilitation in patients with stroke and atrial fibrillation and even those study results were conflicting $[8,10]$. Considering the high prevalence of atrial fibrillation in patients with stroke, it is important to determine the relationship between atrial fibrillation and stroke progress.

In this study, we examined the frequency of atrial fibrillation in patients who participated in a comprehensive rehabilitation program after hospitalization and studied the importance of atrial fibrillation in the rehabilitation process of stroke. Finally, we evaluated the effects of atrial fibrillation on a measure of stroke progress.

\section{MATERIALS AND METHODS}

\section{Subjects}

The subjects of this study were 101 patients who were admitted to the rehabilitation ward in our hospital for comprehensive treatment after onset of cerebral infarction. The rehabilitation continued for approximately 3 months although the rehabilitation schedule differed depending on the condition of the patients (Af group $=91.1 \pm 22.6$ days vs. non-Af group $=80.6 \pm 22.0$ days).
The final diagnosis of all patients was made with computed tomography or magnetic resonance imaging. The inclusion criteria were 1) man or woman aged 40 or more, 2) patient with first onset of cerebral infarction, and 3) patients who were transferred to our unit from the Neurology Department after an acute phase treatment. Among them, 14 patients could not finish the rehabilitation program due to associated systemic diseases, such as pneumonia, sepsis, acute myocardial infarction, acute renal failure, and shock. Except these 14 patients, 87 patients (77 men, 10 women) with stroke were included. Their average age was 72.6 years.

The exclusion criteria were 1) previous stroke history, 2) previous rehabilitation history, 3) concomitant disease that could prevent the rehabilitation program, 4) patients with cerebral hemorrhage, such as subarachnoid and epidural hemorrhages, 5) history of neurological or mental disease or alcohol addiction, and 6) history of paroxysmal atrial fibrillation.

This study was a retrospective study and study data were obtained through medical records. It was conducted with the informed consent of all subjects.

\section{Methods}

The rehabilitation program was done for 30 minutes per session, 5 days per week, for 12 weeks. It consisted of neurodevelopmental treatment, therapeutic exercise, functional electrical stimulation, occupation therapy, dysphagia rehabilitation, physical modalities, art therapy, and psychological counseling.

At the first assessment, subjects were examined regarding risk factors of cerebrovascular disease, diabetes, hypertension, dyslipidemia, smoking, coronary artery disease, congestive heart failure, and valvular heart disease. Atrial fibrillation was defined as having an irregular QRS wave with no visible P wave on a 12-lead ECG or atrial flutter concomitant with irregular ventricular response at the time of admission $[15,16]$.

Motor function was evaluated in terms of the manual muscle test (MMT). Cognitive function was assessed in terms of Mini-Mental State Examination (MMSE-K) scores. The National Institutes of Health Stroke Scale (NIHSS) was used to evaluate the patients' neurological scale. The infarcted lesions were also classified by their locations.

The extent and the type of the cerebral infarction were 
assessed using the Chaudhuri classification [17]. This system is described as follows:

Group I: Normal

Group II: Small superficial infarct involving one cerebral lobe

Group III: Large superficial infarct involving two or more lobes of one cerebral hemisphere

Group IV: Deep infarct; infarct of the internal capsule or basal ganglia

Group V: Combination of deep infarct and large superficial infarct

Group VI: Bihemispheric infarct

The functional activity level was assessed in terms of the scores of the Functional Independence Measure (FIM) for daily living activities at the time of admission and after completion of the rehabilitation program. FIM is a standardized assessment tool, widely used for disability assessments. Its validity and reliability have been demonstrated in previous studies [18-20]. The Modified Barthel Index (MBI) was also used. The MBI is used to measure performance in activities of daily living (ADL) in patients with chronic disease; it evaluates the patient's independence through direct observation of the ADL of the patient along with an interviews. Furthermore, the PULSES profile was used to monitor and predict functional improvement after the rehabilitation program. The score is known to have a high correlation with the FIM and MBI scores [21,22]. At the times of admission and discharge, FIM, MBI, and PULSES scores of patients were determined so that improved functional performance was assessed by comparing the scores between admission and discharge after the rehabilitation program.

\section{Statistical analyses}

Risk factors between patients with and without atrial fibrillation were compared. If the variance according to Levene test was the same, Student t-test was used to conduct a univariate comparison between the two groups of continuous variables. The $\chi^{2}$ test was used for univariate comparisons of nominal variables. A multiple linear regression model was used to analyze the effect of the program on the FIM, MBI, and PULSES scores. The multiple logistic regression model included factors that could be confounding variables, such as age, gender, hospitalization period, time taken from stroke onset to rehabilitation, valvular heart disease, congestive heart failure, and coronary artery disease, including nominal variables that were found to be statistically significantly different between patients with and without atrial fibrillation from the $\chi^{2}$ test, to examine and quantify the relationship between the variables and atrial fibrillation independently. The significance level of the two-sided test was set to $\mathrm{p}<0.05$. The SPSS ver. 17 for Windows (SPSS Inc., Chicago, IL, USA) was used for statistical analyses.

\section{RESULTS}

\section{Characteristics of the subjects}

Among 87 patients with cerebral infarction, only 20 (22.9\%) had atrial fibrillation at the time of admission. The frequency of atrial fibrillation increased as age increased. For example, in patients under 70 years, it was $6.1 \%$ while in patients over 70 years, it was $33.3 \%$.

The average age of patients with atrial fibrillation was $75.1 \pm 5.1$ years while the average age of patients without atrial fibrillation was $71.8 \pm 8.8$ years. The difference was not statistically significant ( $\mathrm{p}=0.116$ ). No significant difference was seen in average morbidity period, diabetes, hypertension, dyslipidemia, smoking rate, or carotid atherosclerosis between the groups.

It took $11.5 \pm 10.7$ days to transfer patients with atrial fibrillation from the Neurological Department that diagnosed cerebral infarction to the Department of Rehabilitation Medicine, while it took 10.5 \pm 8.3 days for patients without atrial fibrillation. Patients with atrial fibrillation had a longer hospitalization period but it was not statistically significant $(\mathrm{p}=0.067)$ (Table 1$)$.

In the univariate comparison, patients with atrial fibrillation had significantly higher prevalence of valvular heart disease, coronary artery disease, and heart failure than patients without atrial fibrillation $\left(\chi^{2}=10.818\right.$ and $\mathrm{p}=0.004, \chi^{2}=10.703$ and $\mathrm{p}=0.002$, and $\chi^{2}=4.656$ and $\mathrm{p}=$ 0.046 , respectively) (Table 1 ).

When factors, such as age, gender, hospitalization period, time taken from stroke onset to rehabilitation, diabetes, hypertension, dyslipidemia, smoking rate, and carotid atherosclerosis, were included in the multiple logistic regression analysis, atrial fibrillation showed a significant correlation with coronary artery disease (odds ratio $[\mathrm{OR}]=5.594,95 \%$ confidence interval $[\mathrm{CI}]=1.592-32.843$ ) and valvular heart disease $(\mathrm{OR}=4.104,95 \% \mathrm{CI}=1.122$ 15.020), while no correlation was seen with congestive 
Table 1. Comparison of clinical characteristics and risk factors in the Af and non-Af groups

\begin{tabular}{|lccccc}
\hline \multirow{2}{*}{ Characteristic } & Af group & Non-Af group & \multicolumn{2}{c}{ Univariate analysis } & \multirow{2}{*}{ p-value } \\
\cline { 1 - 1 } & & & $\chi^{2}$ & t & \\
\hline Age (yr) & $75.1 \pm 5.1$ & $71.8 \pm 8.8$ & & 1.588 & 0.116 \\
Gender (male:female) & $18: 2$ & $59: 8$ & 2.630 & & 0.189 \\
\hline Disease duration (day) & $11.5 \pm 10.7$ & $10.5 \pm 8.3$ & & 0.705 & 0.336 \\
\hline Affected side & & & & \\
\hline Right & 12 & 35 & & & 0.615 \\
\hline Left & 8 & 32 & 0.374 & 0.067 \\
\hline Bilateral & 0 & 0 & & 1.852 & 0.446 \\
\hline LOS (day) & $91.1 \pm 22.6$ & $80.6 \pm 22.0$ & & & 0.611 \\
\hline Diabetes mellitus & $8(40.0)$ & $35(52.2)$ & 0.923 & 0.790 \\
\hline Hypertension & $12(60.0)$ & $34(50.7)$ & 0.529 & & 0.605 \\
\hline Dyslipidemia & $14(70.0)$ & $43(64.2)$ & 0.231 & & $0.046^{*}$ \\
\hline Smoking & $14(70.0)$ & $42(62.7)$ & 0.359 & & $0.004^{*}$ \\
\hline Congestive heart failure & $5(25.0)$ & $5(7.5)$ & 4.656 & & $0.002^{*}$ \\
\hline Valvular heart disease & $6(30.0)$ & $3(4.5)$ & 10.818 & 0.740 \\
\hline Coronary artery disease & $10(50.0)$ & $10(14.9)$ & 10.703 & & \\
\hline Carotid atherosclerosis & $16(80.0)$ & $56(83.6)$ & 0.139 & & \\
\hline
\end{tabular}

Values are presented as mean \pm standard deviation or number (\%).

Af, atrial fibrillation; LOS, length of hospital stay.

${ }^{*} \mathrm{p}<0.05$.

Table 2. Results of multiple logistic regression analysis predicting the relationship between Af and other clinical variables

\begin{tabular}{|lcccc}
\hline & $\beta$ & SE & p-value & R \\
\hline Age & 0.089 & 0.055 & 0.103 & 0.020 \\
\hline Gender & 0.571 & 0.380 & 0.999 & 0.121 \\
\hline LOS & -0.011 & 0.017 & 0.943 & -0.005 \\
\hline Disease duration & 0.005 & 0.031 & 0.871 & 0.000 \\
\hline Coronary heart disease & 1.812 & 0.662 & $0.008^{*}$ & 0.318 \\
\hline Congestive heart failure & 0.209 & 1.200 & 0.862 & 0.080 \\
\hline Valvular heart disease & 1.722 & 1.146 & $0.035^{*}$ & 0.141 \\
\hline Diabetes mellitus & 0.038 & 0.675 & 0.955 & 0.011 \\
\hline Hypertension & 0.251 & 0.772 & 0.153 & 0.065 \\
\hline Smoking & 0.360 & 0.651 & 0.956 & 0.074 \\
\hline Dyslipidemia & 0.510 & 0.742 & 0.491 & 0.088 \\
\hline Carotid atherosclerosis & -0.385 & 0.899 & 0.668 & -0.074 \\
\hline Constant & -4.237 & 1.756 & 0.026 & - \\
\hline
\end{tabular}

Af, atrial fibrillation; LOS, length of hospital stay; SE, standard error.

${ }^{*} \mathrm{p}<0.05$. 
heart failure (Table 2).

Assessment of motor function, cognitive function, and neurological scale

Patient motor function was evaluated with the MMT. Cognitive function was assessed using the MMSE-K. Neurological status before rehabilitation was assessed using the NIHSS. Regarding motor function, the nonAf group had better upper and lower extremity muscle power than the Af group before rehabilitation. However, there was no statistically significant difference between the groups ( $p>0.05)$.

In MMSE- $K$, mild cognitive impairment was detected in both groups. The total score of the non-Af group was better than that of Af group. However, there was no statistically significant difference (Af group $=20.5 \pm 8.3$ vs. non-Af group=23.2 $\pm 7.6 ; \mathrm{p}=0.214$ ). In neurological status using NIHSS, the non-Af group showed a statistically significantly better result in the movement of lower extremities (Af group $=2.4 \pm 1.3$ vs. non-Af group $=0.7 \pm 0.2$; $\mathrm{p}=0.015$ ). However, there was no statistically significant difference in the total score (Af group $=17.5 \pm 6.8$ vs. nonAf group=11.2 $\pm 5.3 ; \mathrm{p}=0.101$ ).

In conclusion, there was no significant difference between the groups in terms of motor function, cognitive function, or neurological scale. In a univariate analysis including each item, there was no significant difference. In a multivariate analysis including each item, statistically significant differences remained, although the influence of atrial fibrillation on the FIM score at discharge decreased a little (MMT: $\mathrm{B}=-8.48, \mathrm{SE}(\mathrm{B})=3.16, \mathrm{p}=0.013$; MMSE-K: $B=-6.22, S E(B)=2.13, p=0.010$; NIHSS: $B=-7.39$, $\mathrm{SE}(\mathrm{B})=2.54, \mathrm{p}=0.009)$. In a multivariate analysis includ-

Table 3. Distribution of ischemic cerebral lesions according to the Chaudhuri classification

\begin{tabular}{cccc}
\hline Group & Af group & Non-Af group & p-value* \\
\hline I & $0(0)$ & $0(0)$ & 1 \\
II & $3(15.0)$ & $18(26.9)$ & 0.257 \\
III & $4(20.0)$ & $13(19.4)$ & 0.905 \\
IV & $9(45.0)$ & $26(38.8)$ & 0.280 \\
V & $4(20.0)$ & $10(14.9)$ & 0.628 \\
VI & $0(0)$ & $0(0)$ & 1 \\
\hline
\end{tabular}

Values are presented as number (\%).

Af, atrial fibrillation.

${ }^{*} \mathrm{p}<0.05$. ing all three items, the difference due to atrial fibrillation was still statistically significant $(B=-5.87, S E(B)=2.01$, $\mathrm{p}=0.027$ ). In a multivariate analysis including each item, a statistically significant differences in MBI score at discharge due to atrial fibrillation remained (MMT: $\mathrm{B}=-6.92$, $\mathrm{SE}(\mathrm{B})=3.27, \mathrm{p}=0.028$; MMSE-K: $\mathrm{B}=-7.96, \mathrm{SE}(\mathrm{B})=4.05$, $\mathrm{p}=0.021$; NIHSS: $\mathrm{B}=-5.38, \mathrm{SE}(\mathrm{B})=2.74, \mathrm{p}=0.033)$. In a multivariate analysis including all three items, the difference of MBI score due to atrial fibrillation was still statistically significant $(B=-5.19, \mathrm{SE}(\mathrm{B})=2.52, \mathrm{p}=0.031)$. In a multivariate analysis including each item, there was a statistically significant difference in PULSES score at discharge due to atrial fibrillation (MMT: $\mathrm{B}=-2.38, \mathrm{SE}(\mathrm{B})=1.04, \mathrm{p}=0.021$; MMSEK: $\mathrm{B}=-2.07, \mathrm{SE}(\mathrm{B})=0.74, \mathrm{p}=0.032$; NIHSS: $\mathrm{B}=-1.75$, $\mathrm{SE}(\mathrm{B})=0.59, \mathrm{p}=0.039)$. In a multivariate analysis including all three items, the difference in PULSES score due to atrial fibrillation remained statistically significant $(\mathrm{B}=1.01, \mathrm{SE}(\mathrm{B})=0.49, \mathrm{p}=0.043)$.

\section{Assessment of brain lesions}

Because location, type, extent of infarcted lesions can affect the results of rehabilitation, we classified the infarcted lesions according to the Chaudhuri classification and by their locations. Regarding the relationship between functional recovery and the location and the extent of cerebral infarction, it is known that patients whose brain lesions are located in the brainstem rather than the cerebral hemispheres or in a cerebral subcortical area rather than a cerebral cortical area, or only in a

Table 4. Comparison of lesion site between Af and nonAf groups

\begin{tabular}{llcc}
\hline \multicolumn{1}{c}{ Location } & Af group & non-Af group & p-value* \\
\hline Temporal lobe & $1(5.0)$ & $5(7.5)$ & 0.884 \\
Frontal lobe & $0(0)$ & $3(4.5)$ & 0.361 \\
Parietal lobe & $2(10.0)$ & $7(10.4)$ & 0.985 \\
Occipital lobe & $0(0)$ & $3(4.5)$ & 0.372 \\
Basal ganglia & $9(45.0)$ & $19(28.4)$ & 0.087 \\
Thalamus & $4(20.0)$ & $15(22.4)$ & 0.792 \\
Brain stem & $3(15.0)$ & $8(11.9)$ & 0.638 \\
Cerebellum & $1(5.0)$ & $7(10.4)$ & 0.518 \\
\hline
\end{tabular}

Values are presented as number (\%).

Only one main lesion was marked in group III lesions in Table 3.

Af, atrial fibrillation.

${ }^{*} \mathrm{p}<0.05$. 
restricted area, such as the putamen or thalamus, among the cerebral subcortical areas, show better results [23-27]. The results are shown in Tables 3 and 4 . According to the Chaudhuri classification, group IV or V (deep infarct or combination of deep infarct and large superficial infarct) lesions were more prevalent in the Af group than the non-Af group. In the non-Af group, group II (small superficial infarct involving one cerebral lobe) lesions were detected more frequently. However, there was no statistically significant difference in the type or extent of brain lesions between the groups according to the Chaudhuri classification. Comparing the locations in both groups, there were more basal ganglia infarctions in the Af group, but the difference was not significant $(\mathrm{p}=0.087)$. In other areas of the brain, there was no statistically significant difference in the distribution of infarcted lesions between the groups. In the Af group, there were more brain lesions located in the brainstem and basal ganglia (including the putamen) that are known to show better results in rehabilitation compared with the non-Af group. However, the functional recovery results after rehabilitation were better in the non-Af group, seemingly contrary to the supposedly unfavorable brain lesions. Thus, location, type, and

Table 5. Admission and discharge FIM, MBI, and PULSES scores in the Af and non-Af groups

\begin{tabular}{|cccc|}
\hline & Af group & Non-Af group & p-value \\
\hline At admission & & & \\
\hline FIM & $62.4 \pm 21.0$ & $76.6 \pm 21.2$ & $0.010^{*}$ \\
\hline MBI & $53.4 \pm 19.7$ & $66.8 \pm 23.8$ & $0.024^{*}$ \\
\hline PULSES & $15 \pm 1.3$ & $11 \pm 2.2$ & $0.035^{*}$ \\
\hline At discharge & & & \\
\hline FIM & $67.8 \pm 20.9$ & $85.2 \pm 21.4$ & $0.002^{*}$ \\
\hline MBI & $57.9 \pm 19.2$ & $72.9 \pm 24.0$ & $0.012^{*}$ \\
\hline PULSES & $13.8 \pm 1.4$ & $8.5 \pm 2.8$ & $0.017^{*}$ \\
\hline Gain & & & \\
\hline FIM & $5.4 \pm 2.4$ & $8.7 \pm 4.1$ & $0.001^{*}$ \\
\hline MBI & $4.5 \pm 2.7$ & $6.0 \pm 3.7$ & $0.048^{*}$ \\
\hline PULSES & $1.2 \pm 0.5$ & $2.5 \pm 1.2$ & $0.001^{*}$ \\
\hline
\end{tabular}

Values are presented as mean \pm standard deviation.

Af, atrial fibrillation; FIM, Functional Independence Measure; MBI, Modified Barthel Index; PULSES, an acronym for six functions and factors (physical condition, upper limb function, lower limb function, sensory function, excretory function, support factors). ${ }^{*} \mathrm{p}<0.05$. extent of infarcted lesions did not seem to affect rehabilitation outcomes in this study.

\section{Functional improvement assessment}

Patients with atrial fibrillation had significantly lower FIM, MBI, and PULSES scores at the time of admission to the Department of Rehabilitation Medicine (62.4 \pm 21.0 vs. $76.6 \pm 21.2, \mathrm{p}=0.010 ; 53.4 \pm 19.7$ vs. $66.8 \pm 23.8, \mathrm{p}=0.024$; and $15 \pm 1.3$ vs. $11 \pm 2.2, \mathrm{p}=0.035$, respectively) (Table 5). Patients with atrial fibrillation also had significantly lower FIM, MBI, and PULSES scores after completion of the rehabilitation program $(67.8 \pm 20.9$ vs. $85.2 \pm 21.4$, $\mathrm{p}=0.002 ; 57.9 \pm 9.2$ vs. $72.9 \pm 24.0, \mathrm{p}=0.012$; and $13.8 \pm 1.4$ vs. $8.5 \pm 2.8, \mathrm{p}=0.017$, respectively) (Table 5). Patients with atrial fibrillation also had significantly lower increases in FIM, MBI, and PULSES scores, obtained by subtracting the scores after the rehabilitation program from those before the program $(5.4 \pm 2.4$ vs. $8.7 \pm 4.1, \mathrm{p}=0.001 ; 4.5 \pm 2.7$ vs. $6.0 \pm 3.7, \mathrm{p}=0.048$; and $1.2 \pm 0.5$ vs. $2.5 \pm 1.2, \mathrm{p}=0.001$, respectively) (Table 5). In the multiple linear regression analysis, atrial fibrillation correlated with a decrease of 11.34 in the FIM score at the time of discharge $(B=-11.34$, $\mathrm{SE}(\mathrm{B})=3.87, \mathrm{p}=0.002)$, of 10.81 in the MBI score at the time of discharge $(B=-10.81, \operatorname{SE}(B)=4.02, p=0.012)$, and of 2.47 in the PULSES score at the time of discharge $(B=-2.47, S E(B)=0.85, p=0.017)$. The effect of atrial fibrillation on the FIM score at the time of discharge was further reduced if the FIM score at the time of admission was included in the analysis. However, it still showed a statistically significant difference $(B=-6.51, S E(B)=2.12$, $\mathrm{p}=0.012$ ). The MBI scores also showed that the difference between atrial fibrillation and the MBI scores at the time of discharge was reduced further if the MBI scores at the time of admission were included in the analysis, but it too still showed a significant correlation $(B=-5.21$, $\mathrm{SE}(\mathrm{B})=2.56, \mathrm{p}=0.035)$. The PULSES scores also showed that the difference between atrial fibrillation and the PULSES scores at the time of discharge was reduced further if the PULSES scores at the time of admission was included in the analysis, but it too still showed a significant correlation $(B=-1.05, \operatorname{SE}(B)=0.56, p=0.041)$.

\section{DISCUSSION}

Atrial fibrillation is the most prevalent arrhythmia in the elderly and is a known major risk factor for cerebral 
infarction. Although it is important to identify the role of atrial fibrillation in the etiology of cerebral infarction, it is also important to assess the effects of atrial fibrillation on prognosis, long-term risk of recurrence of disease, mortality rate, and functional activity level. In this study, the effects of atrial fibrillation on functional activity levels was assessed with regard to patients with cerebral infarction following the acute phase after taking a rehabilitation program. The frequency of atrial fibrillation in the patients of this study was $22.9 \%$, similar to other reports [8-10]. Generally, most previous studies reported that patients with cerebral infarction and atrial fibrillation were older than patients with cerebral infarction and sinus rhythm. However, in our study, no significant difference in age was found between the groups. However, a group with atrial fibrillation had a higher average age and also higher prevalence of atrial fibrillation as age increased.

The functional activity level of two groups, based on FIM, MBI, and PULSES scores, showed that patients without atrial fibrillation had better results even before the rehabilitation program. Patients with atrial fibrillation showed functional recovery after the rehabilitation program but their recovery was not as high as in patients without atrial fibrillation. At the end of the rehabilitation program, patients with atrial fibrillation showed statistically significantly higher disability and lower function activity performance than patients without atrial fibrillation.

Compared with patients with sinus rhythm, patients with atrial fibrillation had a higher probability of coronary artery disease, valvular heart disease, and congestive heart failure. However, the prevalence of other risk factors, such as diabetes, hypertension, smoking, dyslipidemia, and carotid atherosclerosis, showed no significant difference between the groups. Such concomitant diseases in the patients with atrial fibrillation could contribute to or be the reason for the lower functional activity performance. Previously, Roth [28] reported that the presence of heart diseases, such as ischemic heart disease and congestive heart failure, negatively affected the rehabilitation of stroke patients, their long-term followup progress, and treatment.

This study result showed that atrial fibrillation was related not only to a higher risk of cerebral infarction occurrence but also higher disabilities in patients with cerebral infarction. Thus, it can be concluded that atrial fibrillation has a negative effect on recovery from cerebral infarction. Many previous studies reported that atrial fibrillation was related to a higher death rate in stroke patients; in particular, it was closely related to death rate after 30 days from the onset of stroke $[8,11,12,29,30]$. Only a few studies have examined the relationship of atrial fibrillation with functional results in the process of recovery after stroke $[8,10]$. Most of these reports explained that the high death rate and low functional activity performance in patients with atrial fibrillation were due to older age, larger brain lesions, and more severe initial neurological disabilities.

Although there was no significant difference in age, motor function, cognitive function, neurological scale, or infarcted brain lesions between the groups, patients with and without atrial fibrillation did show significant differences in functional activity performance. This result indicates that factors other than age, pretreatment motor and cognitive functions, neurological scale and infarcted brain lesions contributed to the recovery from cerebral infarction. For example, differences in brain lesions, concomitant diseases, and hemodynamic factors in the recovery process from cerebral infarction, and the will of the patient and family are also considered to be contributing factors.

A limitation of this study is that it was difficult to assess the degree of neurological disability of patients who were admitted to the Department of Neurology at the time of admission because this study chose subjects who were transferred from the Department of Neurology after acute phase treatment for the cerebral infarction. It was also difficult to assess the severity of the functional disability according to a morbidity period of atrial fibrillation.

Also, we did not classify the cerebral infarction by pathophysiology or by vascular territory. The Af group was mainly embolic infarction and the non-Af group was mainly thrombotic or lacunar infarctions. Additionally, there were patients with multifocal infarctions in which two or more or vascular territories were involved. Thus, it was difficult to compare rehabilitation outcomes of cerebral infarction according to the classification by pathophysiology or by vascular territories, which is why we used the Chaudhuri classification. Lack of long-term follow-up and the retrospective study design are also limitations of this study.

In conclusion, atrial fibrillation can be considered as a 
negative prognostic factor in terms of functional progress in patients with cerebral infarction. Thus, more attention to heart assessments and treatments should be given to patients with cerebral infarction and atrial fibrillation. It would be helpful for the active participation of patients with atrial fibrillation in the rehabilitation program to recognize the presence of atrial fibrillation and monitor their heart problems attentively during the rehabilitation treatment. Thus, active treatment following an assessment of the underlying diseases and structural diseases of the heart that can generate atrial fibrillation can prevent the occurrence and recurrence of cerebral infarction as well as increase the success rate of rehabilitation programs.

\section{CONFLICT OF INTEREST}

No potential conflict of interest relevant to this article was reported.

\section{REFERENCES}

1. Shibazaki K, Kimura K, Aoki J, Saji N, Sakai K. Early initiation of new oral anticoagulants in acute stroke and TIA patients with nonvalvular atrial fibrillation. J Neurol Sci 2013;331:90-3.

2. Feinberg WM, Blackshear JL, Laupacis A, Kronmal R, Hart RG. Prevalence, age distribution, and gender of patients with atrial fibrillation. Analysis and implications. Arch Intern Med 1995;155:469-73.

3. Kannel WB, Wolf PA, Benjamin EJ, Levy D. Prevalence, incidence, prognosis, and predisposing conditions for atrial fibrillation: population-based estimates. Am J Cardiol 1998;82:2N-9N.

4. Wolf PA, Abbott RD, Kannel WB. Atrial fibrillation as an independent risk factor for stroke: the Framingham Study. Stroke 1991;22:983-8.

5. Petersen P. Thromboembolic complications in atrial fibrillation. Stroke 1990;21:4-13.

6. Go AS. The epidemiology of atrial fibrillation in elderly persons: the tip of the iceberg. Am J Geriatr Cardiol 2005;14:56-61.

7. Wolf PA, Dawber TR, Thomas HE Jr, Kannel WB. Epidemiologic assessment of chronic atrial fibrillation and risk of stroke: the Framingham study. Neurology 1978;28:973-7.
8. Lin HJ, Wolf PA, Kelly-Hayes M, Beiser AS, Kase CS, Benjamin EJ, et al. Stroke severity in atrial fibrillation: the Framingham Study. Stroke 1996;27:1760-4.

9. Kaarisalo MM, Immonen-Raiha P, Marttila RJ, Salomaa V, Kaarsalo E, Salmi K, et al. Atrial fibrillation and stroke: mortality and causes of death after the first acute ischemic stroke. Stroke 1997;28:311-5.

10. Sandercock P, Bamford J, Dennis M, Burn J, Slattery J, Jones L, et al. Atrial fibrillation and stroke: prevalence in different types of stroke and influence on early and long term prognosis (Oxfordshire community stroke project). BMJ 1992;305:1460-5.

11. Candelise L, Pinardi G, Morabito A. Mortality in acute stroke with atrial fibrillation. The Italian Acute Stroke Study Group. Stroke 1991;22:169-74.

12. Gustafsson C, Britton M. Pathogenetic mechanism of stroke in non-valvular atrial fibrillation: follow-up of stroke patients with and without atrial fibrillation. J Intern Med 1991;230:11-6.

13. Fisher CM. Reducing risks of cerebral embolism. Geriatrics 1979;34:59-61, 65-6.

14. Krahn AD, Manfreda J, Tate RB, Mathewson FA, Cuddy TE. The natural history of atrial fibrillation: incidence, risk factors, and prognosis in the Manitoba Follow-Up Study. Am J Med 1995;98:476-84.

15. Gonzalez Toledo ME, Klein FR, Riccio PM, Cassara FP, Munoz Giacomelli F, Racosta JM, et al. Atrial fibrillation detected after acute ischemic stroke: evidence supporting the neurogenic hypothesis. J Stroke Cerebrovasc Dis 2013;22:e486-91.

16. Fuster V, Ryden LE, Cannom DS, Crijns HJ, Curtis AB, Ellenbogen KA, et al. ACC/AHA/ESC 2006 Guidelines for the Management of Patients with Atrial Fibrillation: a report of the American College of Cardiology/ American Heart Association Task Force on Practice Guidelines and the European Society of Cardiology Committee for Practice Guidelines (Writing Committee to Revise the 2001 Guidelines for the Management of Patients With Atrial Fibrillation): developed in collaboration with the European Heart Rhythm Association and the Heart Rhythm Society. Circulation 2006;114:e257-354.

17. Chaudhuri G, Harvey RF, Sulton LD, Lambert RW. Computerized tomography head scans as predictors of functional outcome of stroke patients. Arch Phys Med Rehabil 1988;69:496-8. 
18. Granger CV, Cotter AC, Hamilton BB, Fiedler RC. Functional assessment scales: a study of persons after stroke. Arch Phys Med Rehabil 1993;74:133-8.

19. Hamilton BB, Laughlin JA, Fiedler RC, Granger CV. Interrater reliability of the 7 -level functional independence measure (FIM). Scand J Rehabil Med 1994;26:115-9.

20. Shin YI, Kim YH, Seo JH, Kim YC. The influencing factors on FIM score in the patients with stroke. J Korean Acad Rehabil Med 1996;20:833-8.

21. Granger CV, Albrecht GL, Hamilton BB. Outcome of comprehensive medical rehabilitation: measurement by PULSES profile and the Barthel Index. Arch Phys Med Rehabil 1979;60:145-54.

22. Marshall SC, Heisel B, Grinnell D. Validity of the PULSES profile compared with the Functional Independence Measure for measuring disability in a stroke rehabilitation setting. Arch Phys Med Rehabil 1999;80:760-5.

23. Kim KT, Kang MJ, Lee HN, An JD, Cho C, Bae J. Clinical factors associated with functional status at discharge in stroke patients. J Korean Acad Rehabil Med 2003;27:300-8.

24. Chen CL, Tang FT, Chen HC, Chung CY, Wong MK. Brain lesion size and location: effects on motor recov- ery and functional outcome in stroke patients. Arch Phys Med Rehabil 2000;81:447-52.

25. Chua KS, Kong KH. Functional outcome in brain stem stroke patients after rehabilitation. Arch Phys Med Rehabil 1996;77:194-7.

26. Kong KH, Chua KS, Tow AP. Clinical characteristics and functional outcome of stroke patients 75 years old and older. Arch Phys Med Rehabil 1998;79:1535-9.

27. Miyai I, Suzuki T, Kang J, Volpe BT. Improved functional outcome in patients with hemorrhagic stroke in putamen and thalamus compared with those with stroke restricted to the putamen or thalamus. Stroke 2000;31:1365-9.

28. Roth EJ. Heart disease in patients with stroke. Part II: Impact and implications for rehabilitation. Arch Phys Med Rehabil 1994;75:94-101.

29. Arboix A, Garcia-Eroles L, Comes E, Oliveres M, Targa C, Balcells M, et al. Importance of cardiovascular risk profile for in-hospital mortality due to cerebral infarction. Rev Esp Cardiol 2008;61:1020-9.

30. Paciaroni M, Agnelli G, Ageno W, Caso V, Corea F, Lanari A, et al. Risk factors for cerebral ischemic events in patients with atrial fibrillation on warfarin for stroke prevention. Atherosclerosis 2010;212:564-6. 